\title{
On normality, ethnicity, and missing values in quantitative trait locus mapping
} Aurélie Labbe* ${ }^{* 1}$ and Hanna Wormald ${ }^{\dagger 2}$

Address: ${ }^{1}$ Département de Mathématiques et de Statistiques, Université Laval, Québec, G1K7P4, QC, Canada and 2Department of Statistics and Actuarial Sciences, University of Waterloo, 200 University Avenue West, Waterloo N2L3G1, ON, Canada

Email: Aurélie Labbe* - alabbe@math.uwaterloo.ca; Hanna Wormald - hdwormald@uwaterloo.ca

* Corresponding author †Equal contributors

from Genetic Analysis Workshop 14: Microsatellite and single-nucleotide polymorphism

Noordwijkerhout, The Netherlands, 7-10 September 2004

Published: 30 December 2005

BMC Genetics 2005, 6(Suppl I):S52 doi:I0.1186/147I-2156-6-SI-S52

\begin{abstract}
Background: This paper deals with the detection of significant linkage for quantitative traits using a variance components approach. Microsatellite markers were obtained for the Genetic Analysis Workshop 14 Collaborative Study on the Genetics of Alcoholism data. Ethnic heterogeneity, highly skewed quantitative measures, and a high rate of missing values are all present in this dataset and well known to impact upon linkage analysis. This makes it a good candidate for investigation.

Results: As expected, we observed a number of changes in LOD scores, especially for chromosomes I, 7, and 18, along with the three factors studied. A dramatic example of such changes can be found in chromosome 7. Highly significant linkage to one of the quantitative traits became insignificant when a proper normalizing transformation of the trait was used and when analysis was carried out on an ethnically homogeneous subset of the original pedigrees.

Conclusion: In agreement with existing literature, transforming a trait to ensure normality using a Box-Cox transformation is highly recommended in order to avoid false-positive linkages. Furthermore, pedigrees should be sorted by ethnic groups and analyses should be carried out separately. Finally, one should be aware that the inclusion of covariates with a high rate of missing values reduces considerably the number of subjects included in the model. In such a case, the loss in power may be large. Imputation methods are then recommended.
\end{abstract}

\section{Background}

The purpose of this paper is to illustrate the impact of three factors: robustness to non-normality, population admixture, and covariates with a high rate of missing values, on the linkage detected using a variance components approach. The effect of these factors has been well studied in linkage analysis. As we shall see, the Collaborative Study on the Genetics of Alcoholism (COGA) dataset offers a very good illustration of the dramatic changes observed when such aspects are not considered carefully.
Variance components approaches determine whether genetic variation at a specific chromosomal location can explain the variation in the phenotype [1]. This nonparametric approach is based on the difference of LOD score of the likelihood under the null and alternative hypothesis, where likelihoods are computed under a multivariate normality assumption of the trait under study. This method is known to have optimal power when the model is well specified [2] but is also known as lacking robustness to the normality assumption of the trait [3]. 
Population admixture is another phenomenon that has been studied [4]. It is known, for example, that the difference in the allele frequencies or disease rates between subpopulations may lead to violation of the key assumptions of Hardy-Weinberg equilibrium (HWE) and linkage equilibrium (LE) between markers. For example, as Grigul et al. [5] showed, linkage results can be greatly affected by clustering families from an admixed population using pedigree-specific marker allele frequencies.

The last aspect considered in this work deals with the inclusion of covariates in the model with a high proportion of missing values. Several methods of imputation for missing values are available, and we refer for example to Fridley et al. [6] regarding the polygenic model. Despite this fact, many genetic analysis packages still ignore individuals with missing covariates in the model. This can have a very strong impact on the results, because the sample size is considerably reduced, affecting the power of the analysis in most cases.

\section{Methods}

Genome-wide scan analysis was performed on 329 microsatellite markers obtained for 1,350 of the 1,614 individuals in the Genetic Analysis Workshop 14 (GAW14) COGA pedigree data.

\section{Selecting the quantitative traits}

We first sought candidate traits that could be used as an illustration of the impact of the factors studied. After performing genome-wide scan analyses on different electrophysiological quantitative phenotypes, we selected three traits, named ttth1, ttdt 3 , and ttdt 4 in the COGA data, that expressed significant linkage in some regions of the genome. These traits are also commonly referred to as event-related potential (ERP) traits.

\section{Preparing the pedigrees}

In order to measure the influence of ethnicity of pedigrees on quantitative trait locus (QTL) linkage detection, we ran a genome-wide scan analysis on two sets of COGA pedigrees. The first set contained all pedigrees as in the GAW14 COGA data (1,614 individuals distributed in 143 families) and the second set contained 105 pedigrees extracted from the initial set of pedigrees, namely those whose members claimed ethnicity $=6$ (non-Hispanic White, 1,237 individuals). These last pedigrees are called the White pedigrees through this paper. Note that some pedigrees were truncated in order to preserve the unity of the self-reported ethnicity.

\section{Detecting linkage}

All the statistical linkage analyses were carried out using the software SOLAR (Sequential Oligogenic Linkage Analysis Routines) [1,7], which determines whether genetic variation at a specific chromosomal location can explain the variation in the phenotype. This statistical method is an extension of the strategy developed by Amos [8].

Following the variance components method, a quantitative genetic analysis with covariate screening was performed. The best model was chosen by iteratively adding the covariates to the model, and by estimating the different parameters, such as the total additive genetic heritability (H2r) and the covariates regression coefficients, by maximum likelihood. Only significant covariates were kept in the model.

Identity-by-descent (IBD) probabilities and multipoint identity-by-descent (MIBD) matrices (for multipoint analysis) were computed using allele frequencies either provided with the COGA data (not ethnicity-specific) or estimated by SOLAR using a maximum likelihood approach (as in the White pedigree case). In multipoint linkage analysis, the Kosambi map function was used as supplied with the GAW14 data.

\section{Transformation of the trait to ensure normality}

Preparing the quantitative trait for QTL analysis is a crucial step. Normality of the trait is the basic requirement of the statistical method we used. It is important to ensure that the empirical distribution of the trait considered follows this requirement. Skewness and kurtosis are good measures that allow the identification of a potential violation of the normality assumption. Skewness is a measure of the asymmetry of the distribution while kurtosis is an indicator of how close the distribution matches a bell shape. If the distribution is normal, both measures should be zero. A common approach in regression-type models uses the logarithmic transformation. Such a transformation is often effective in reducing skewness and kurtosis. More generally, a Box-Cox transformation (see [9]) can be used in order to maximize the closeness to normality for the transformed data. Data is then transformed to a power $\lambda$, where $\lambda$ is chosen to be optimal. In the case of the COGA dataset, a value $\lambda=1 / 4$ was found to be optimal for the traits tth 1 , ttdt 3 , and ttdt 4 , regardless of the type of pedigree used.

\section{Covariates}

If not specified otherwise, the covariates included in the polygenic model (after a screening of all the covariates) involving the transformed trait and the White pedigree were sex, erpage (age at ERP examination), ALDX11 (alcohol dependency) for trait ttth $1^{1 / 4}$, erpage, ALDX12 for trait ttdt $3^{1 / 4}$ and erpage, ALDX12 for trait ttdt $4^{1 / 4}$.

Covariates were taken as provided in the COGA dataset, except in the case of ALDX1, ALDX2 that were transformed into a number of indicator variables in order to 
Table I: QTL LOD score, per pedigree and per type of transformation

\begin{tabular}{|c|c|c|c|c|c|c|}
\hline \multirow{3}{*}{$\begin{array}{c}\text { Chr } \\
\text { I }\end{array}$} & \multicolumn{2}{|c|}{ Trait [skewness; kurtosis] } & \multicolumn{4}{|c|}{ Maximum LOD score and location (cM) } \\
\hline & \multirow[b]{2}{*}{$\mathrm{ttdt} 3$} & \multirow[b]{2}{*}[0.57;0.25]{} & \multicolumn{2}{|c|}{ Whole pedigree } & \multicolumn{2}{|c|}{ White pedigree } \\
\hline & & & 1.4 & $(65 \mathrm{cM})$ & 1.07 & $(65 \mathrm{cM})$ \\
\hline & $\ln (\mathrm{ttdt} 3)$ & {$[-0.2 ; 0.083]$} & 2.79 & (64 cM) & 2.24 & $(64 \mathrm{cM})$ \\
\hline & $\operatorname{ttdt} 31 / 4$ & {$[-0.0006 ;-0.076]$} & 2.37 & $(63$ cM) & 2.03 & $(65 \mathrm{cM})$ \\
\hline \multirow[t]{3}{*}{1} & $\operatorname{ttdt} 4$ & {$[0.55 ; 0.129]$} & 1.14 & $(67$ cM) & 1.2 & $(69 \mathrm{cM})$ \\
\hline & $\ln (\operatorname{ttdt} 4)$ & {$[-0.23 ; 0.19]$} & 2.29 & $(65$ cM) & 2.02 & $(67 \mathrm{cM})$ \\
\hline & $\operatorname{ttdt} 4^{1 / 4}$ & {$[-0.01 ;-0.069]$} & 1.99 & $(66$ cM) & 1.86 & $(66 \mathrm{cM})$ \\
\hline \multirow[t]{3}{*}{18} & $\operatorname{ttdt} 3$ & {$[0.57 ; 0.25]$} & 3.63 & (54 cM) & 3.05 & $(54 \mathrm{cM})$ \\
\hline & $\ln (\operatorname{ttdt} 3)$ & {$[-0.2 ; 0.083]$} & 4.2 & (54 cM) & 4.2 & $(54 \mathrm{cM})$ \\
\hline & $\operatorname{tttdt} 3^{1 / 4}$ & {$[-0.0006 ;-0.076]$} & 4.67 & (54 cM) & 3.8 & (55 cM) \\
\hline \multirow[t]{3}{*}{18} & $\operatorname{ttdt} 4$ & {$[0.55 ; 0.129]$} & 1.6 & (53 cM) & 1.69 & $(55 \mathrm{cM})$ \\
\hline & $\ln (\operatorname{ttdt} 4)$ & {$[-0.23 ; 0.19]$} & 2.26 & $(53$ cM) & 2.26 & (53 cM) \\
\hline & $\operatorname{ttdt} 4^{1 / 4}$ & {$[-0.01 ;-0.069]$} & 2.39 & (49 cM) & 2.29 & (52 cM) \\
\hline \multirow[t]{3}{*}{7} & ttthl & {$[0.62 ; 0.27]$} & 4.08 & $(\mathrm{I} 54 \mathrm{cM})$ & 3.49 & $(160 \mathrm{cM})$ \\
\hline & $\ln (\mathrm{ttth} I)$ & {$[-0.25 ; 0.55]$} & 3.55 & $(161 \mathrm{cM})$ & 2.67 & $(160 \mathrm{cM})$ \\
\hline & $\left.\operatorname{ttth}\right|^{1 / 4}$ & {$[-0.0053 ; 0.156]$} & 3.87 & $(161 \mathrm{cM})$ & 1.19 & $(112 \mathrm{cM})$ \\
\hline
\end{tabular}

use them as categorical covariates. For instance, ALDX12 represents $A L D X 1=5$ (affected) and $A L D X 12=0$ represents $A L D X 1<5$.

\section{Results}

\section{Linkage detection}

Pedigree-based analyses demonstrated three QTLs on chromosome 1 (between markers D1S1598 and D1S2134; 1p34-1p33), chromosome 18 (between markers D18A535 and D18A877), and chromosome 7 (between markers D7S1804 and D7S509; 7q32-7q34). The first two QTLs appear to be linked to the traits labelled ttdt 3 and ttdt 4 . As mentioned in Table 1, the corresponding LOD scores range from 1.07 up to 4.67 , depending on the type of transformation used on the traits as well as the set of pedigrees.

Findings on chromosome 7 show a strong linkage signal for the other measure, $\mathrm{tth} 1$, with a maximum LOD score of 4.08. However, this linkage seems to be strongly related to the self-reported ethnicity of the pedigrees as well as the skewness and kurtosis of the trait.

\section{Influence of the non-normality of the trait}

Figure 1 shows the LOD scores across the loci for the traits ttth $1, \ln (\mathrm{ttth} 1)$, and $\mathrm{tth} 1^{1 / 4}$, on chromosome 7 for the White pedigree. Dramatic changes in the LOD scores can be observed when different transformations are applied. The trait ttth1 in its original form appears to be strongly linked to a QTL around $160 \mathrm{cM}(\mathrm{LOD}=3.49)$. The loga- rithmic transformation of this trait gives only suggestive linkage to the same QTL (LOD = 2.67). However, the BoxCox transformation of $\mathrm{tth} 1$, i.e., $\mathrm{tth} 1^{1 / 4}$, does not show any linkage result at this locus (LOD $=0.06)$. A maximum LOD score of 1.19 is obtained at location $112 \mathrm{cM}$. In this case, not only has the significance of the LOD peak has been dramatically reduced, but it has also been moved by $48 \mathrm{cM}$.

Clearly, such changes are not systematic across traits, chromosomes, and pedigrees. However, we illustrate here an extreme situation. Table 1 gives information on changes to LOD scores according to the transformation used on traits ttth 1 , ttdt 3 , and $\operatorname{ttdt} 4$ for chromosomes 1 , 7 , and 18. This table illustrates a number of different changes in LOD scores when transformations are applied. From this it can be observed that an appropriate transformation on the trait (normality improved) causes changes in the linkage detected (increases of LOD score, as in chromosome 1 and 18, or decreases, as in chromosome 7).

\section{Influence of ethnicity}

Table 1 also illustrates the impact of the segregation of the pedigrees by ethnic groups. Overall linkage findings appeared to be slightly more significant when ethnic admixture is present (note however that the size of population is increased by $30 \%$ in this case). Another effect of ethnicity admixture can be noticed in chromosome 7 for trait ttth1. The fact that a QTL is detected and preserved along the transformations in the whole pedigrees only 


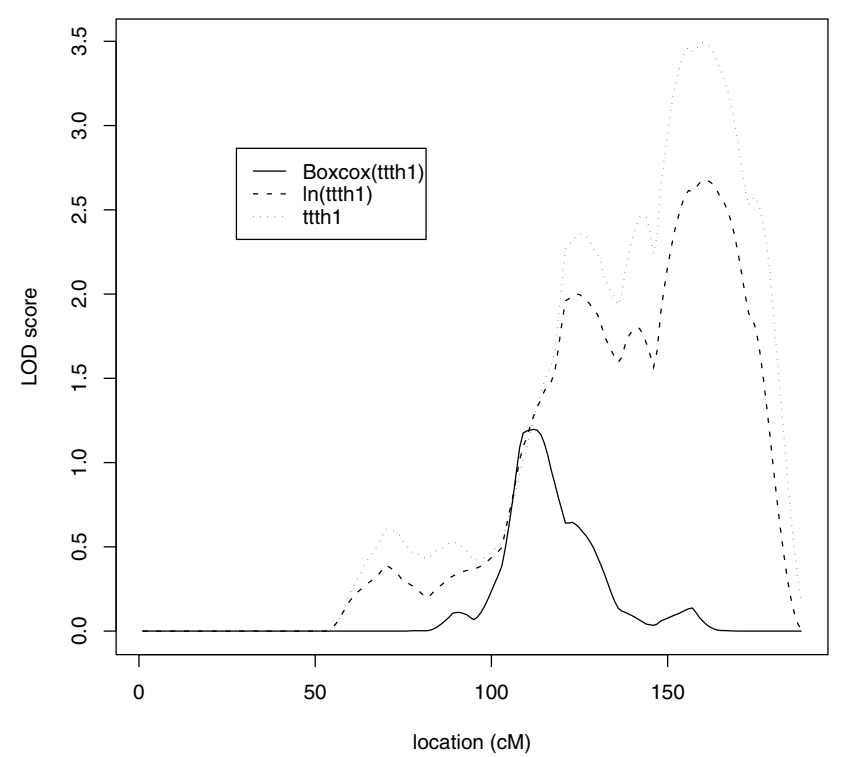

Figure I

LOD scores for chromosome 7 (White pedigree): transformations of the trait ttthl.

may suggest that it occurs in reality mainly in the nonWhite pedigrees. Due to the small number of these families available and due to their ethnic heterogeneity, we could not confirm this hypothesis by a statistical analysis.

\section{Adding more covariates in the polygenic model}

As an illustration of the impact that the addition of covariates has in the linkage detected, we study the case of chromosome 1 using the White pedigrees and the trait ttdt $3^{1 /}$ 4 . Figure 2 presents the changes in LOD scores when different covariates are added in the polygenic model. Note that the optimal model includes two variables, erpage and $A L D X 12$, with a total of 680 individuals. If the variable cigpkyrs (number of packs of cigarettes per day for one year) is also included in the model (for a total of 445 individuals), we observe a deviation from the optimal model, regarding the significance of the LOD score, along the chromosome. If the variable cigpkyrs is replaced by the variable ageonset 1 , which contains $59.5 \%$ of missing values, the number of subjects included in the model drops to 348 individuals and we can observe extreme changes in the significance of the LOD score.

\section{Discussion and conclusion}

As we have seen, the COGA dataset provides a very clear illustration of the effects that ethnicity, covariates, and the normality of the trait have on the linkage observed. One should be aware that mixing different ethnic groups may introduce some noise, leading to the failure to detect strong linkage that may be present in one or more of the

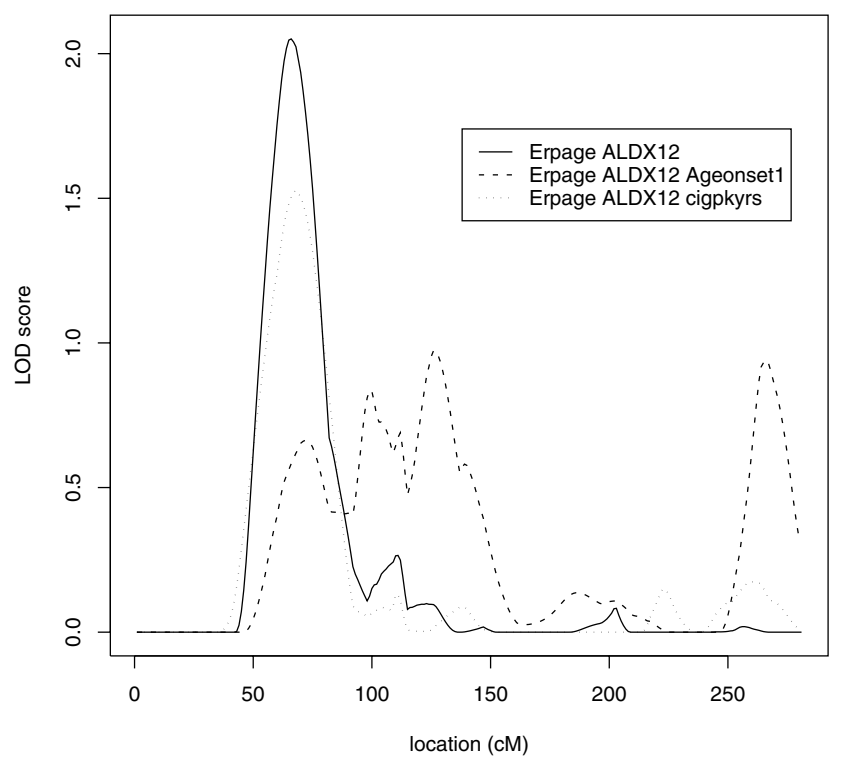

Figure 2

LOD scores for chromosome I (White pedigree) using different polygenic models on the trait ttdt $3^{1 / 4}$.

groups. As a result, populations admixtures should be avoided if no evidence suggests that the different ethnic groups behave similarly in terms of the trait and markers considered. Note that in the case studied, the preliminary multivariate analysis of the traits considered showed highly significant segregation by the self-reported ethnicity.

When adding covariates in the model, one should pay special attention to the trade-off between the gain of information due to the covariate and the loss of information due to the reduction of the sample size. If possible, imputation methods should be considered.

Finally, any statistical models are built on data assumptions, such as the normality of the trait. Again, being aware of these assumptions and trying to guarantee their validity is key in the success of an analysis. Note that many other factors could be discussed, such as the impact of a violation of some other model assumptions such as Hardy-Weinberg equilibrium and linkage equilibrium. The type of map used is also crucial in fine mapping studies. Some inconsistencies in the maps lead to great differences in the location of the linkage detected, especially when high density SNPs are used.

\section{Abbreviations}

COGA: Collaborative Study on the Genetics of Alcoholism 
ERP: Event-related potential

GAW: Genetic Analysis Workshop

HWE: Hardy-Weinberg equilibrium

IBD: Identity by descent

LE: Linkage equilibrium

MIBD: Multipoint identity by descent

QTL: Quantitative trait locus

\section{Authors' contributions}

Both authors contributed equally to this work in all the aspects of the paper.

\section{Acknowledgements}

The authors thank Professor Mary E. Thompson, Department of Statistics and Actuarial Sciences at the University of Waterloo, for her very helpful comments and suggestions, as well as her administrative and financial support.

\section{References}

I. Almasy L, Blangero J: Multipoint quantitative-trait linkage analysis in general pedigrees. Am J Hum Genet 1998, 62:1 198-121 I.

2. Feingold $\mathrm{E}$ : Methods for linkage analysis of quantitative trait loci in humans. Theor Popul Biol 200I, 60:167-180.

3. Allison DB, Neale MC, Zanolli R, Schork NJ, Amos Cl, Blangero J: Testing the robustness of the likelihood ratio test in a variance component quantitative-trait loci-mapping procedure. Am J Hum Genet 1999, 65:53I-544.

4. Barnholtz-Sloan JS, de Andrade M, Chakraborty R: The impact of population admixture on traditional linkage analysis. Ethn Dis 200I, II:519-53I.

5. Grigul J, Alexandrova R, Paterson AD: Clustering of pedigrees using marker allele frequencies: impact on linkage analysis. Genet Epidemiol 200I, 2 I (Suppl. I):S6I-S66.

6. Fridley B, Rabe K, de Andrade M: Imputation methods for missing data for polygenic models. BMC Genetics 2003, 4:S42.

7. Blangero J, Almasy L: Multipoint oligogenic linkage analysis of quantitative-traits. Genet Epidemiol 1997, 14:959-964.

8. Amos Cl: Robust variance-component approach for assessing genetic linkage in pedigrees. Am J Hum Genet 1994, 54:535-543.

9. Venable WN, Ripley BD: Modern Applied Statistics with S Fourth edition. New York: Springer; 2002.

\section{Publish with Bio Med Central and every scientist can read your work free of charge}

"BioMed Central will be the most significant development for disseminating the results of biomedical research in our lifetime. "

Sir Paul Nurse, Cancer Research UK

Your research papers will be:

- available free of charge to the entire biomedical community

- peer reviewed and published immediately upon acceptance

- cited in PubMed and archived on PubMed Central

- yours - you keep the copyright

Submit your manuscript here:

http://www.biomedcentral.com/info/publishing_adv.asp 